\title{
A physically based model of temperature and strain rate dependent yield in BCC metals: Implementation into crystal plasticity
}

\author{
Hojun Lim ${ }^{\mathrm{a}, *}$, Corbett C. Battaile ${ }^{\mathrm{a}}$, Jay D. Carroll ${ }^{\mathrm{a}}$, Brad L. Boyce ${ }^{\mathrm{a}}$, Christopher R. Weinberger ${ }^{\mathrm{b}}$ \\ ${ }^{a}$ Sandia National Laboratories, P.O. Box 5800, Albuquerque NM, 87185 \\ ${ }^{b}$ Department of Mechanical Engineering and Mechanics, Drexel University, Philadelphia, PA, 19104
}

\begin{abstract}
In this work, we develop a crystal plasticity finite element model (CP-FEM) that constitutively captures the temperature and strain rate dependent flow stresses in pure BCC refractory metals. This model is based on the kink-pair theory developed by Seeger (Seeger, 1981) and is calibrated to available data from single crystal experiments to produce accurate and convenient constitutive laws that are implemented into a BCC crystal plasticity model. The model is then used to predict temperature and strain rate dependent yield stresses of single and polycrystal BCC refractory metals (molybdenum, tantalum, tungsten and niobium) and compared with existing experimental data. To connect to larger length scales, classical continuum-scale constitutive models are fit to the CP-FEM predictions of polycrystal yield stresses. The results produced by this model, based on kink-pair theory and with origins in dislocation mechanics, show excellent agreement with the Mechanical Threshold Stress (MTS) model for temperature and strain-rate dependent flow. This framework provides a method to bridge multiple length scales in modeling the deformation of BCC metals.
\end{abstract}

Keywords: Kink-pair theory, Temperature, Strain rate, Molybdenum, Tantalum, Tungsten, Niobium

\section{Introduction}

Body-centered-cubic (BCC) metals are well-known to exhibit not only strong temperature and strain rate dependent flow stresses, but also yield behavior that violates Schmid's Law (Christian, 1983; Taylor, 1992; Duesbery and Vitek, 1998; Weinberger et al., 2012; Lim et al., 2013b). Most

\footnotetext{
${ }^{*}$ Corresponding author

Email address: hnlim@sandia.gov (Hojun Lim)
} 
other close-packed metals, most notably those with face-centered-cubic (FCC) crystal structure, do not share these characteristics. The strong temperature and strain rate dependent flow stress results from the thermally activated motion of screw dislocations (Seeger, 1981, 2001; Argon, 2008; Butt, 2007) and the non-Schmid effects are generally associated with the distortion of the dislocation core structures (Christian, 1983; Ito and Vitek, 2001; Gröger et al., 2008).

Dislocation kink-pair theory (Seeger, 1981, 2001; Butt, 2007; Argon, 2008) has been used not only to model the thermally activated motion of screw dislocations in $\mathrm{BCC}$ metals, but also to successfully describe the strong temperature and strain rate dependent flow stresses of various BCC single crystals, e.g. molybdenum (Hollang et al., 1997, 2001), tantalum (Werner, 1987), tungsten (Brunner, 2000) and niobium (Seeger and Holzwarth, 2006). Variations of kink-pair theory in BCC transition metals are well-documented in the review by Weinberger et al. (Weinberger et al., 2013a). Since these models describe dislocation-level mechanisms, they are amenable to incorporation in crystal plasticity models.

Crystal plasticity finite element models (CP-FEM) have been widely used to predict mechanical response and texture evolution in polycrystal deformation (Peirce et al., 1982; Asaro, 1983; Dawson, 2000). Most of these are specific to FCC metals, which exhibit far less dependence of the flow stress on temperature and strain rate as compared to BCC metals. Although most FCC viscoplasitc constitutive models invoke a formulation that involves the rate of slip on individual slip systems (Peirce et al., 1982; Asaro and Needleman, 1985), this is primarily a numerical device, and thus inappropriate for describing the physical temperature and strain rate dependence of the flow stress of BCC metals. Thus, the strain rate and temperature effects must be accounted for in the constitutive formulations capturing these effects explicitly. In particular, BCC transition metals are widely used in many high strain rate and high temperature applications where these effects are of paramount importance.

Continuum constitutive models, e.g. Johnson-Cook (JC) (Johnson and Cook, 1983, 1985), Zerilli-Armstrong (ZA) (Zerilli and Armstrong, 1987) and Mechanical Threshold Stress (MTS) (Follansbee and Kocks, 1988), treat these effects in regimes of high temperatures and strain rates. These models have successfully reproduced temperature and strain rate behaviors of BCC polycrystals such as Armco iron (Johnson and Cook, 1983, 1985; Zerilli and Armstrong, 1987), steel alloys (Johnson and Cook, 1983, 1985), molybdenum (Nemat-Nasser et al., 1999; Cheng et al., 2001), 
tungsten and tungsten alloys (Johnson and Cook, 1983; Lennon and Ramesh, 2000), tantalum and tantalum alloys (Zerilli and Armstrong, 1990; Chen and Gray, 1996; Nemat-Nasser and Isaacs, 1997; Nemat-Nasser and Guo, 2000a; Park et al., 2011), niobium (Nemat-Nasser and Guo, 2000a) and vanadium (Nemat-Nasser and Guo, 2000b). However, some models, e.g. JC, are empirical formulations that lack detailed physical basis and only serve as a convenient fit to experimental data. In contrast, the ZA and MTS models are developed based on the thermal activation of dislocations, but these models are usually parameterized using data from bulk polycrystals and thus are not applicable to crystallographic descriptions of deformation, e.g. crystal plasticity, that are commonly used to describe deformation behavior at the scale of the microstructure.

In this work, we analyze kink-pair theory and compare its predictions to measured single crystal tensile data from the literature (Werner, 1987; Hollang et al., 1997, 2001; Brunner, 2000; Seeger and Holzwarth, 2006) to provide temperature and strain rate dependent flow stresses of tantalum, tungsten, molybdenum and niobium. Physically-based single crystal constitutive equations, based on dislocation kink-pair theory, are implemented into a BCC finite element crystal plasticity model (Weinberger et al., 2012; Lim et al., 2013b) to predict temperature and strain rate dependent yielding of single and polycrystal molybdenum, tantalum, tungsten and niobium. Furthermore, the CP-FEM results are used to parameterize polycrystal continuum constitutive models. This capability provides a convenient constitutive formulation for approximating the effects of temperature and strain rate on plastic deformation of BCC metals at the grain scale that is based on fundamental dislocation physics of atomic-scale unit processes, and is consistent with behavior at the continuum/ macroscale.

\section{Kink-Pair Theory}

Dislocation-based kink-pair theory describes the temperature and strain rate dependent flow stresses in BCC metals (Seeger, 1981, 2001; Butt, 2007; Argon, 2008) by relating the stress required to move a dislocation over the Peierls potential, $\tau_{p}$, to the temperature, $T$, and applied strain rate, $\dot{\gamma}$. The theory is based on the assumption that the flow stress, $\tau$, resolved onto the active slip system is composed of thermal and athermal contributions as follows :

$$
\tau(T, \dot{\gamma})=\tau^{*}(T, \dot{\gamma})+\bar{\tau}
$$


where $\tau^{*}$ is the thermal component of the flow stress while $\bar{\tau}$ represents the athermal stress that is independent of strain rate and weakly dependent on temperature through the shear modulus. $\bar{\tau}$ accounts for long-range interactions such as the effects of forest dislocations, impurities, solutes and grain boundaries; and can be determined using measured values of the resolved shear stress above a critical temperature, $T_{c}(\dot{\gamma})$, where the thermal part of the lattice friction is negligible. Athermal contributions obtained from single crystal experiments are $\bar{\tau}=53 \mathrm{MPa}, 12 \mathrm{MPa}, 27 \mathrm{MPa}$ and 18 MPa for molybdenum, tungsten, tantalum and niobium, respectively (Hollang et al., 2001; Brunner, 2000; Werner, 1987; Werner and Seeger, 1988).

Following the work of Seeger (Seeger, 1981, 2001, 1995), there are three regimes in the flow stress behavior as a function of temperature. In the high temperature regime, above the critical temperature, $T_{c}(\dot{\gamma})$, the thermal dependence is similar to that of FCC metals. Below the critical temperature, $T_{c}(\dot{\gamma})$, BCC metals exhibit thermally activated flow that deviates from the behavior of FCC metals, and the mobility of screw dislocations is the main contribution to the high resistance to flow. Below $T_{c}(\dot{\gamma})$, there are two regimes that are divided by $\hat{T}(\dot{\gamma})$. In Regime I, where $\hat{T}(\dot{\gamma})<$ $T<T_{c}(\dot{\gamma})$, the kinks are fully formed and well separated (Figure 1(a)), and the energetics are controlled by the elastic repulsion between kinks. Thus, the kink-pair formation enthalpy, $\Delta H_{k p}^{*}$, can be obtained using the Elastic Interaction (EI) model. Regime II $(T<\hat{T}(\dot{\gamma}))$ is characterized by an activation barrier where the dislocation does not reach the adjacent Peierls valley (Figure 1(b)). In this regime, kinks are not fully formed and can be described by the Line Tension (LT) model. Figures 1 (a) and (b) illustrate the transition states of the kink-pairs for Regime I and Regime II, respectively.

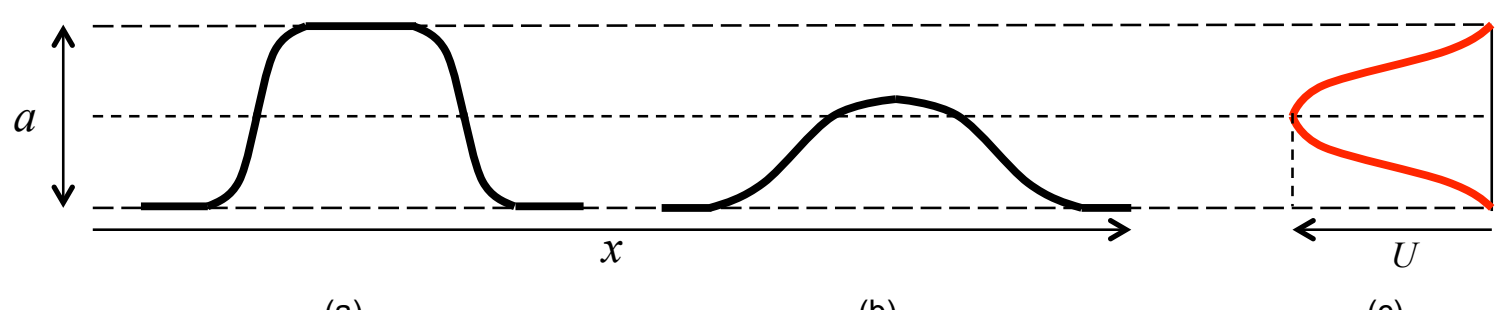

(a)

(b)

(c)

Figure 1: Schematics of (a) the transition state of Regime I where kink-pairs are fully formed, (b) Regime II where the dislocation does not reach the next Peierls valley and (c) Peierls potential. In this schematic, $a$ is the lattice spacing in the direction of dislocation advance, $x$ is the spatial position along a dislocation line length, and $U$ represents the potential energy barrier for dislocation motion. 
An Arrhenius expression relating the activation enthalpy, strain rate and temperature can be obtained by using a transition state theory as follows (Seeger, 1981):

$$
\dot{\gamma}=\dot{\gamma}_{0} \exp \left(-\frac{\Delta H_{k p}^{*}\left(\tau^{*}\right)}{k_{\mathrm{B}} T}\right)
$$

where $\dot{\gamma}_{0}$ is a reference strain rate, $H_{k p}^{*}$ is the double kink activation enthalpy and $k_{\mathrm{B}}$ is the Boltzmann constant. $\dot{\gamma}_{0}$ depends weakly on temperature and, when compared directly to the exponential temperature dependence, can be approximated as a constant.

\subsection{Governing equations for Regime I}

In Regime I, the activation enthalpy associated with the repulsion of a fully-formed kink-pair, $\Delta H_{k p}^{*}$, can be represented using the EI model as follows (Hirth and Lothe, 1982; Seeger, 1981):

$$
\Delta H_{k p}^{*}=2 H_{k}-2 \sqrt{\frac{\mathcal{T} b h^{3}}{2} \tau^{*}}
$$

where $H_{k}$ is the formation enthalpy of an isolated kink, $h$ is the kink height, $b$ is the magnitude of the Burger's vector and $\mathcal{T}$ is the pre-logarithmic dislocation line tension factor of a screw dislocation. Using Equations (2) and (3), a relationship between $\tau^{*}$, temperature and strain rate can be obtained:

$$
\tau^{*}=\tau_{p}^{E I}\left(1-\frac{k_{\mathrm{B}} T \ln \left(\dot{\gamma}_{0} / \dot{\gamma}\right)}{2 H_{k}}\right)^{2}=\tau_{p}^{E I}\left(1-\frac{T}{T_{c}(\dot{\gamma})}\right)^{2}
$$

where $\tau_{p}^{E I}=\frac{2 H_{k}^{2}}{\mathfrak{T} b h^{3}}$ and $T_{c}(\dot{\gamma})=\frac{2 H_{k}}{k_{\mathrm{B}} \ln \left(\dot{\gamma}_{0} / \dot{\gamma}\right)}$. Equation (4) can be fit to single crystal experiments to determine material parameters for the EI model, e.g. $T_{c}(\dot{\gamma}), \tau_{p}^{E I}, 2 H_{k}$, and $\dot{\gamma}_{0}$. For example, a linear fit to measured $\tau^{* 0.5}$ and temperature can be used to obtain $T_{c}(\dot{\gamma})$ and $\tau_{p}^{E I}$ for each strain rate in Regime I. $2 H_{k}$ and $\dot{\gamma}_{0}$ can be obtained by the linear fit between $1 / T_{c}(\dot{\gamma})$ and $\ln (\dot{\gamma})$. Table 1 lists best-fit material constants for the EI model in Regime I for molybdenum, tantalum, tungsten and niobium obtained from fitting to single crystal experiments in the literature (Hollang et al., 1997, 2001; Brunner, 2000; Seeger and Holzwarth, 2006). Note that these best-fit material constants for the EI model agree well with previous studies, except for in tantalum where $2 H_{k}$ is reported to be $0.97 \mathrm{eV}$ (Werner, 1987). 
Table 1: Material parameters for the EI model for molybdenum, tantalum, tungsten and niobium in Regime I.

\begin{tabular}{cccc}
\hline Mat. & $\tau_{p}^{E I}(\mathrm{MPa})$ & $\dot{\gamma}_{0}\left(s^{-1}\right)$ & $2 H_{k}(\mathrm{eV})$ \\
\hline Mo & 1156 & $3.75 \times 10^{9}$ & 1.27 \\
Ta & 406 & $2.99 \times 10^{6}$ & 0.85 \\
$\mathrm{~W}$ & 2035 & $3.71 \times 10^{10}$ & 2.06 \\
$\mathrm{Nb}$ & 576 & $1.14 \times 10^{8}$ & 0.68 \\
\hline
\end{tabular}

\subsection{Governing equations for Regime II}

At low temperatures $(T<\hat{T}), \tau^{* 0.5}$ is not linear in temperature (Equation (4)) and thus the LT model should be used to describe flow in this regime (Regime II). The activation enthalpy of an incompletely formed kink-pair, $\Delta H_{k p}^{*}$, is determined using a simple dislocation line tension model that incorporates the shape of the Peierls potential in the formulation. Analytical expressions that relate the activation enthalpy, applied stress, temperature and strain rate can only be obtained under certain circumstances. Thus, a number of authors have introduced model potentials to facilitate solutions (Seeger, 1981; Argon, 2008; Guyot and Dorn, 1964; Caillard and Martin, 2003; Eshelby, 1962). Notably, a sinusoidal potential has no analytical solution while those comprised of parabolas do (Celli et al., 1963). Some common representations of Peierls potentials are shown in Figure 2.

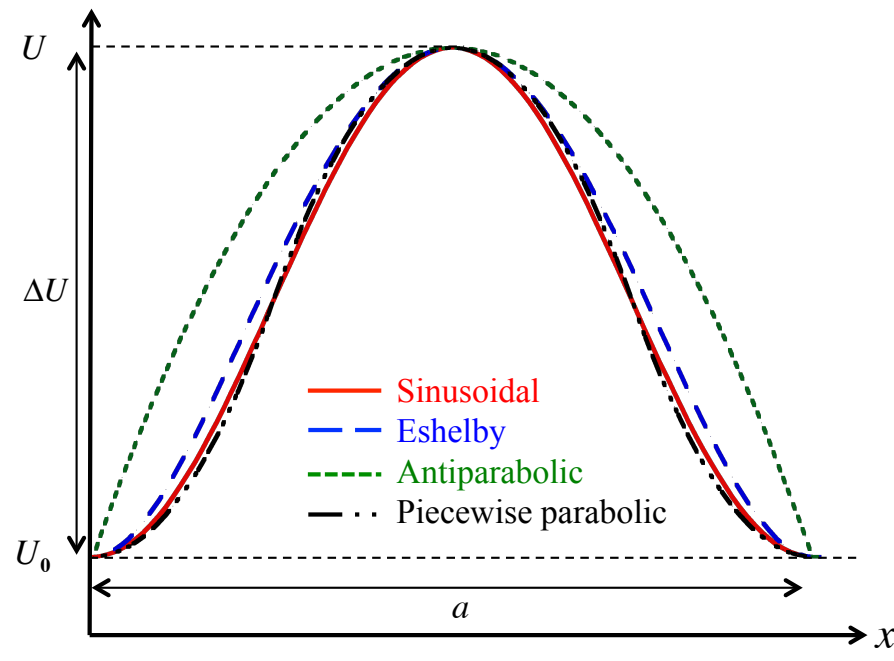

$$
\begin{aligned}
& \text { Sinusoidal } \quad U=U_{0}+\frac{\Delta U}{2}\left(1-\cos \left(\frac{2 \pi x}{a}\right)\right) \\
& \text { Eshelby } \quad U=U_{0}+16 \Delta U\left(\frac{x}{a}\right)^{2}\left(1-\frac{x}{a}\right)^{2} \\
& \text { Antiparabolic } U=U_{0}+4 \Delta U\left(\frac{x}{a}\right)\left(1-\frac{x}{a}\right)
\end{aligned}
$$

Piecewise parabolic

$$
\begin{array}{ll}
x \leq \frac{1}{4} a & U=U_{0}+8 \Delta U\left(\frac{x}{a}\right)^{2} \\
\frac{1}{4} a \leq x \leq \frac{3}{4} a & U=U_{0}+\Delta U\left(a-4\left(a-\frac{1}{2}\right)^{-1}\left(\frac{x}{a}-\frac{a}{2}\right)^{2}\right) \\
\frac{3}{4} a \leq x \leq a & U=U_{0}+8 \Delta U\left(\frac{x}{a}-a\right)^{2}
\end{array}
$$

Figure 2: Different representations of the Peierls potential: Sinusoidal, Eshelby, antiparabolic and piecewise parabolic funtions. 
Following the previous work by Seeger et al. (Seeger, 1981; Werner, 1987; Seeger, 1995; Seeger and Holzwarth, 2006) who adopted an Eshelby potential for this purpose, $\Delta H_{k p}^{*}$ can be approximated as follows:

$$
\Delta H_{k p}^{*} \approx 2 H_{k}-h b \tau^{*} \sqrt{\frac{\mathcal{S}}{U^{\prime \prime}(h)}}\left(1+\ln \frac{\tilde{\tau}}{\tau^{*}}\right)
$$

where $\mathcal{S}$ is the line tension factor of a screw dislocation, $U(h)$ is the Peierls potential and $\tilde{\tau}=$ $2 h U^{\prime \prime}(h) / b$. In addition, $\tilde{\tau}=12 \sqrt{3} \tau_{p}, U^{\prime \prime}(h)=\frac{6 \sqrt{2} b \tau_{p}}{h}$ and $h b \sqrt{\frac{\delta}{U^{\prime \prime}(h)}}=\frac{H_{k}}{\sqrt{3} \tau_{p}}$ (Eshelby, 1962) are satisfied. The activation energy for the Eshelby potential in Equation (5) can be alternatively written as follows (Seeger, 1981):

$$
\Delta H_{k p}^{*}=2 H_{k}\left[1-\frac{\tau^{*}}{2 \sqrt{3} \tau_{p}}\left(1-\ln \frac{\tau^{*}}{12 \sqrt{3} \tau_{p}}\right)+\mathcal{O}\left(\tau^{*}\right)\right]
$$

where $\mathcal{O}\left(\tau^{*}\right)$ is the higher order terms of the expansion. Combining Equation (6) with Equation (2) and ignoring higher order terms, an implicit equation for $\tau^{*}$ is obtained (Seeger, 1981):

$$
\frac{T}{T_{c}(\dot{\gamma})}=1-\frac{\tau^{*}}{2 \sqrt{3} \tau_{p}}\left(1-\ln \frac{\tau^{*}}{12 \sqrt{3} \tau_{p}}\right) \quad \text { Eshelby }
$$

This expression relates the temperature, strain rate (through $T_{c}$ ) and the resolved shear stress; and can be fit to experimental data. This is essentially the procedure used in several previous studies (Seeger, 1981; Werner, 1987; Brunner, 2000; Hollang et al., 1997, 2001; Seeger and Holzwarth, 2006) with minor differences. Note that the above derivation for the Eshelby potential (Seeger, 1981) is valid for low values of $\tau^{*}$ where higher order terms in Equation (6) are ignored. At high stress and low temperatures, $\tau^{*}$ can be approximated as follows (Seeger, 1981; Brunner and Diehl, 1991):

$$
\tau^{*}=\tau_{p}\left(1-\frac{2}{3}\left(\frac{5}{6}\right)^{4 / 5}\left(\frac{T}{T_{c}(\dot{\gamma})}\right)^{4 / 5}\right) \quad \text { Eshelby }\left(\operatorname{high} \tau^{*}\right)
$$

In addition, an empirical formulation that agrees with Equation (7) in the low stress/ high 
temperature regime and Equation (8) in the high stress/ low temperature regime is:

$$
\tau^{*}=\tau_{p}\left(1-\left(\frac{T}{T_{c}(\dot{\gamma})}\right)^{4 / 5}\right)^{5 / 4} \quad \text { Empirical }
$$

Alternatively, the antiparabolic representation of Peierls potential allows more simple and direct representation of the flow stress in Regime II compared to the Eshelby potential (Equation (7)). Using the antiparabolic Peierls potential, $\Delta H_{k p}^{*}$ can be represented as follows (Argon, 2008; Caillard and Martin, 2003; Dorn and Rajnak, 1964):

$$
\Delta H_{k p}^{*}=2 H_{k}\left(1-\frac{\tau^{*}}{\tau_{p}}\right)^{2}
$$

Combining Equation (10) with Equation (2), $\tau^{*}$ is obtained as follows:

$$
\tau^{*}=\tau_{p}\left(1-\sqrt{\frac{k_{B} T \ln \left(\dot{\gamma}_{0} / \dot{\gamma}\right)}{2 H_{k}}}\right)=\tau_{p}\left(1-\left(\frac{T}{T_{c}(\dot{\gamma})}\right)^{1 / 2}\right) \quad \text { Antiparabolic }
$$

Explicit representation of $\tau^{*}$ using the antiparabolic Peierls potential can be readily fit to single crystal stress-strain data in Regime II. In this work, $2 H_{k}$ and $\dot{\gamma}_{0}$ values obtained from the EI model are adopted while $\tau_{p}$ is obtained from a best fit to measured single crystal data. The best-fit $\tau_{p}$ values are $835 \mathrm{MPa}, 320 \mathrm{MPa}, 1038 \mathrm{MPa}$ and $402 \mathrm{MPa}$ for molybdenum, tantalum, tungsten and niobium, respectively.

Figures 3 (a)-(d) show the measured (Hollang et al., 1997; Werner, 1987; Brunner, 2000; Seeger and Holzwarth, 2006) and fitted $\tau^{*}$ using the EI and LT models with the antiparabolic Peierls potential as a function of temperature for various strain rates. Experimentally, $\tau^{*}$ values for single crystal molybdenum, tantalum and niobium were obtained from the Ackermann-Mughrabi technique which uses pre-deformed specimens that are cyclic-saturated to produce very stable dislocation cell structure and thus minimize the variance in the observed response (Ackermann et al., 1983; Werner, 1987; Hollang et al., 1997; Seeger and Holzwarth, 2006). On the other hand, lower yield points from uniaxial tension tests were used to obtain $\tau^{*}$ values for tungsten single crystals (Brunner, 2000).

The EI and LT models in the two temperature regimes accurately reproduce the temperature 

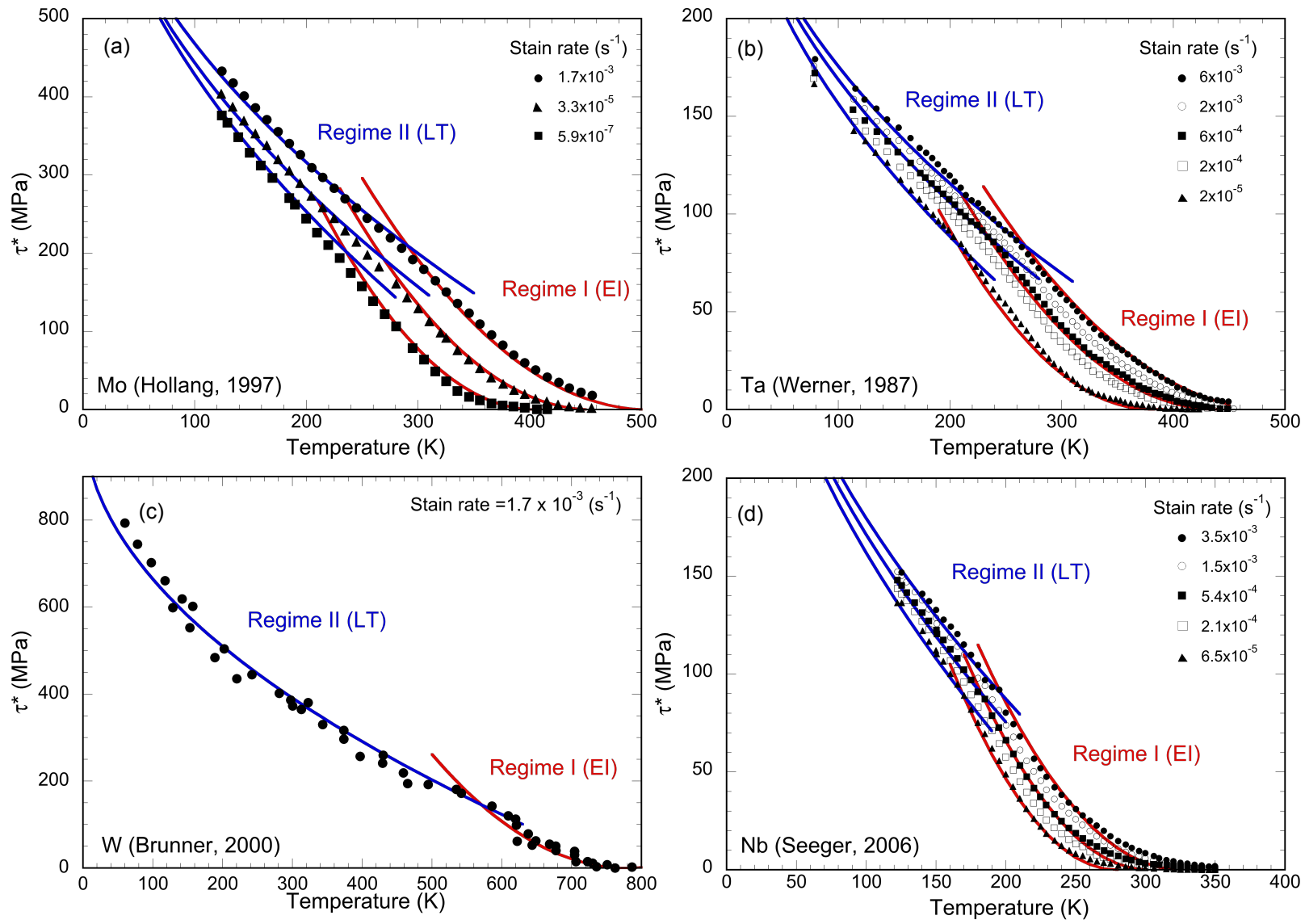

Figure 3: A plot of measured (data points) and fitted (solid lines) $\tau^{*}$ using the EI and LT models for (a) molybdenum, (b) tantalum, (c) tungsten and (d) niobium single crystals. Fits in tantalum and niobium single crystals are for the highest, lowest and middle strain rates. In Regime II, the LT model adopting the antiparabolic Peierls potential is used.

and strain rate dependent flow stresses for all four BCC metals. Note that the transition stresses between two regimes, $\hat{\tau}$, are $180 \mathrm{MPa}, 93 \mathrm{MPa}, 150 \mathrm{MPa}$ and $93 \mathrm{MPa}$ for molybdenum, tantalum, tungsten and niobium, respectively.

Figures 4 (a)-(d) compare the best-fit $\tau^{*}$ in Regime II using the antiparabolic and Eshelby representations of Peierls potential and the empirical formulation (Equations (11), (7) and (9), respectively ). The LT model adopting the antiparabolic representation of the Peierls potential and the empirical formulation reproduce $\tau^{*}$ slightly better than the LT model using the Eshelby potential. Table 2 lists standard deviations between measured and fitted $\tau^{*}$ using the three models in Regime II. The antiparabolic potential predicts measured $\tau^{*}$ of tantalum, tungsten and niobium more accurately than the empirical formulation. Although $\tau^{*}$ obtained from the three models show relatively good agreement with measured data at moderate temperatures, larger deviations in $\tau^{*}$ are 

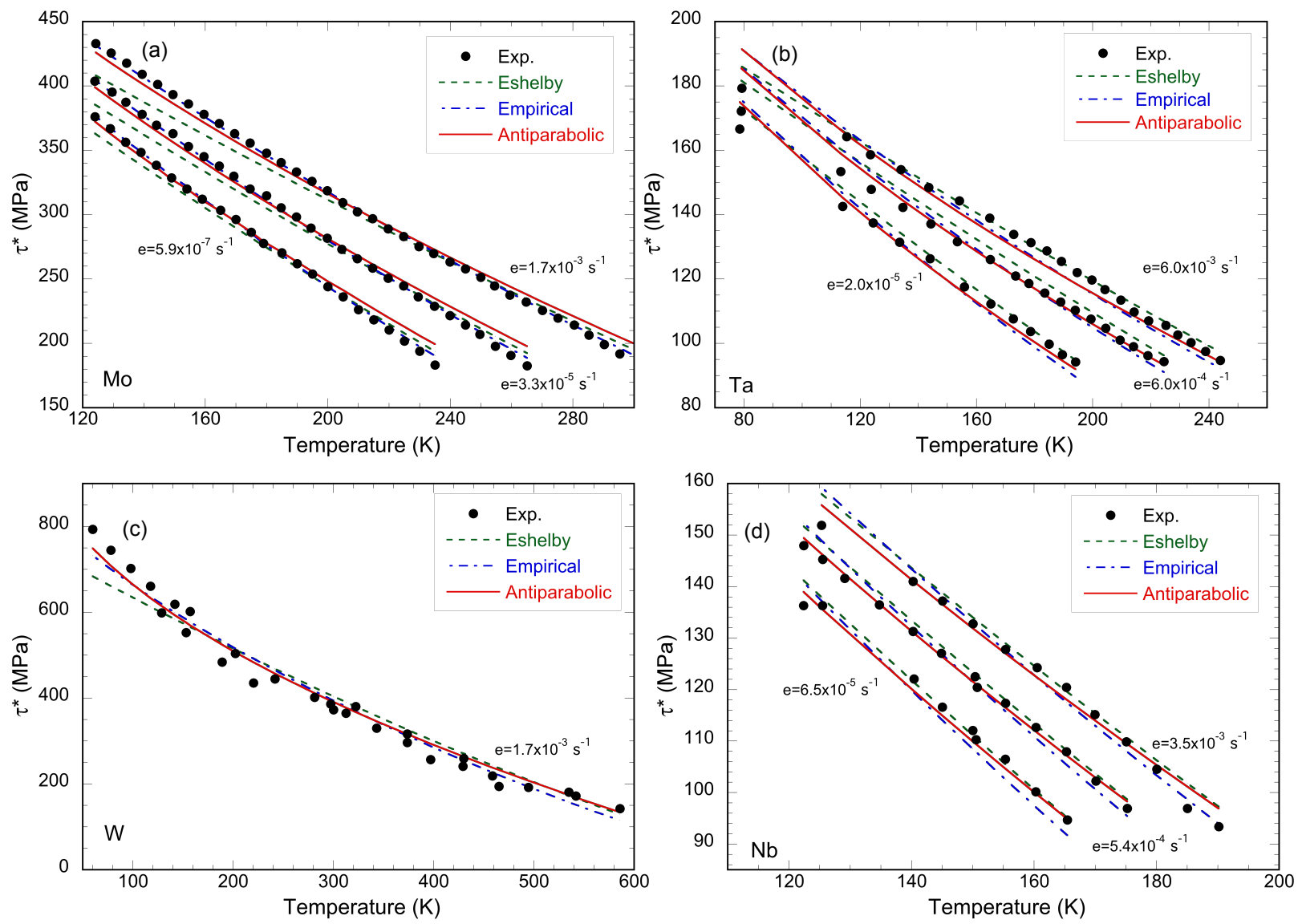

Figure 4: LT models using the Eshelby potential, the antiparabolic potential and the empirical formulation fit to measured data for (a) molybdenum, (b) tantalum, (c) tungsten and (d) niobium.

observed at very low temperatures where the antiparabolic representation of the Peierls potential exhibits higher $\tau^{*}$ for low temperatures and the Eshelby Peierls potential exhibits a nearly linear dependence of $\tau^{*}$ with temperature. As shown in Table 3, the best-fit $\tau_{p}$, representing $\tau^{*}$ at $0 \mathrm{~K}$, is largest for the antiparabolic Peierls potential; this feature enables a more accurate prediction of high $\tau^{*}$ for tungsten at low temperatures.

In order to understand the difference in predicted $\tau^{*}$ with the choice of Peierls potentials, $\Delta H_{k p}^{*} / 2 H_{k}$ versus $\tau^{*} / \tau_{p}$ is plotted using various representations of Peierls potentials and the empirical formulation, as shown in Figure 5. Here, Eshelby and Eshelby (high $\tau^{*}$ ) represent $\tau^{*}$ approximations using Equations (7) and (8), respectively. For the piecewise parabolic function, the potential is parabolic with an upward concave parabola at $y \leq a / 4$ and $3 a / 4 \leq y \leq a$, and a downward concave parabola at $a / 4 \leq y \leq 3 a / 4$. As $\Delta H_{k p}^{*}$ approaches zero, the Eshelby potential 
Table 2: Standard deviations between the measured and fitted $\tau^{*}$ using the Eshelby potential, the antiparabolic potential and the empirical formulation in Regime II. (Unit: MPa)

\begin{tabular}{cccc}
\hline & Eshelby (Eqn.(7)) & Empirical (Eqn.(9)) & Antiparabolic (Eqn.(11)) \\
\hline Mo & 9.7 & 2.1 & 6.3 \\
$\mathrm{Ta}$ & 3.3 & 4.1 & 3.6 \\
$\mathrm{~W}$ & 39.4 & 27.1 & 24.5 \\
$\mathrm{Nb}$ & 2.3 & 2.6 & 1.7 \\
\hline Average & 13.7 & 9.0 & 9.0 \\
\hline
\end{tabular}

Table 3: Best-fit Peierls stresses, $\tau_{p}$, obtained using the Eshelby potential, the antiparabolic potential and the empirical formulation in Regime II. (Unit: MPa)

\begin{tabular}{cccc}
\hline & Eshelby (Eqn.(7)) & Empirical (Eqn.(9)) & Antiparabolic (Eqn.(11)) \\
\hline Mo & 711 & 697 & 835 \\
$\mathrm{Ta}$ & 286 & 267 & 320 \\
$\mathrm{~W}$ & 929 & 871 & 1039 \\
$\mathrm{Nb}$ & 360 & 349 & 410 \\
\hline
\end{tabular}

(Equation (7)) does not converge to the expected value of $\tau^{*}=\tau_{p}$, but instead to approximately $\tau^{*}=0.82 \tau_{p}$. This deviation at high stress regime for Seeger's approximation using the Eshelby potential is due to the truncation of the higher order terms in Equation (6). This not only explains the deviation between calculated $\tau^{*}$ for Eshelby and antiparabolic potentials at low temperatures, but also sheds lights on better agreements at low temperature between the antiparabolic potential and the tungsten data as shown in Figure 4 (c). If we compensate for this and instead directly compare the computed Peierls stress, $\tau_{p}$, between the two models rather than the extrapolated $\tau^{*}$, $\tau_{p}$ for the antiparabolic and the Eshelby potentials, we find they differ by as much as $16 \%$ (for molybdenum) which suggests that there is still some disagreement between the fits. Thus, the LT model adopting an antiparabolic Peierls potential not only provides a more convenient numerical representation of $\tau^{*}$ that can be used in crystal plasticity simulations but also reproduces $\tau^{*}$ more accurately.

As shown in Figure 2, the shape of the piecewise parabolic function is very close to that of the sinusoidal function which was shown to agree well with Peierls potential for molybdenum, tantalum, tungsten and niobium using DFT simulations (Weinberger et al., 2013b). Despite the 


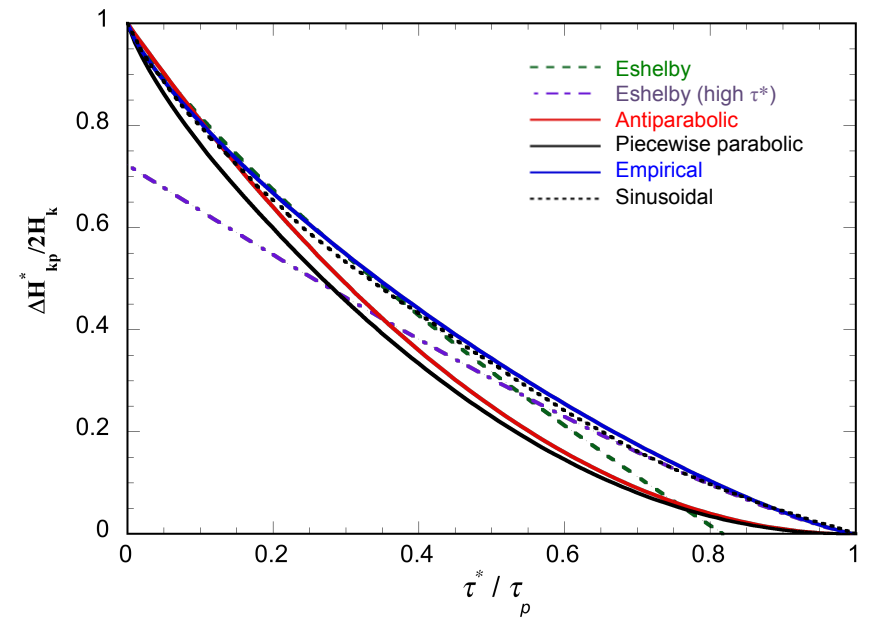

Figure 5: A plot of $\Delta H_{k p}^{*} / 2 H_{k}$ versus $\tau^{*} / \tau_{p}$ using different Peierls potentials.

obvious differences between the antiparabolic and piecewise parabolic representations (Figure 2), the functional forms of $\Delta H_{k p}^{*} / 2 H_{k}$ versus $\tau^{*} / \tau_{p}$ using two representations are quite similar at high $\tau^{*}$ where the LT model is applicable. In a similar manner, the empirical formulation (Equation (9)) agrees very well with the sinusoidal representation of the Peierls potential and agrees with Eshelby approximations, Equations (7) and (8), at low and high stress regimes, respectively (Figure 5). Thus, the empirical representation of the temperature and strain rate dependent flow stress in Equation (9) also provides a simple model for accurately representing the flow behavior of BCC metals.

Based on these comparisons, we advocate the use of either the antiparabolic or the empirical potential for CP-FEM constitutive models. Both accurately model the data at low temperatures and moderate to high stresses using simple analytical forms. One point that may be unsettling with the antiparabolic potential is that the shape is decidedly different from the Eshelby or Sinusoidal and DFT data (Weinberger et al., 2013b). However, the activation energy as a function of stress for the anti-parabolic potential is similar to the piecewise parabolic potential (Figure 5), which has a nearly-sinusoidal shape (Figure 2). Thus, the antiparabolic potential activation energy function can be thought of as an approximation of the piecewise parabolic potential. 
Table 4: The twenty-four $\{110\}$ slip systems.

\begin{tabular}{cc|cc|cc|cc}
\hline$\alpha$ & Slip System & $\alpha$ & Slip System & $\alpha$ & Slip System & $\alpha$ & Slip System \\
\hline 1 & $(01 \overline{1})[111]$ & 7 & $(0 \overline{1} \overline{1})[\overline{1} \overline{1} 1]$ & 13 & $(01 \overline{1})[\overline{1} \overline{1} \overline{1}]$ & 19 & $(0 \overline{1} \overline{1})[11 \overline{1}]$ \\
2 & $(\overline{1} 01)[111]$ & 8 & $(101)[\overline{1} \overline{1} 1]$ & 14 & $(\overline{1} 01)[\overline{1} \overline{1} \overline{1}]$ & 20 & $(101)[11 \overline{1}]$ \\
3 & $(1 \overline{1} 0)[111]$ & 9 & $(\overline{1} 10)[\overline{1} \overline{1} 1]$ & 15 & $(1 \overline{1} 0)[\overline{1} \overline{1} \overline{1}]$ & 21 & $(\overline{1} 10)[11 \overline{1}]$ \\
4 & $(\overline{1} 0 \overline{1})[\overline{1} 11]$ & 10 & $(10 \overline{1})[1 \overline{1} 1]$ & 16 & $(\overline{1} 0 \overline{1})[1 \overline{1} \overline{1}]$ & 22 & $(10 \overline{1})[\overline{1} 1 \overline{1}]$ \\
5 & $(0 \overline{1} 1)[\overline{1} 11]$ & 11 & $(011)[1 \overline{1} 1]$ & 17 & $(0 \overline{1} 1)[1 \overline{1} \overline{1}]$ & 23 & $(011)[\overline{1} 1 \overline{1}]$ \\
6 & $(110)[\overline{1} 11]$ & 12 & $(\overline{1} \overline{1} 0)[1 \overline{1} 1]$ & 18 & $(110)[1 \overline{1} \overline{1}]$ & 24 & $(\overline{1} \overline{1} 0)[\overline{1} 1 \overline{1}]$ \\
\hline
\end{tabular}

\section{Crystal Plasticity Formulation}

The temperature and strain rate dependent flow stress formulations, based on kink-pair theory as described in the preceding section, were implemented into a crystal plasticity model within a finite element code (JAS-3D) developed at Sandia National Laboratories (Biffle, 1987; Lim et al., 2013b). The model is based on a well-established continuum formulation and follows multiplicative decomposition of the deformation gradient (Lee, 1969; Rice, 1971; Hill and Rice, 1972; Peirce et al., 1982; Dingreville et al., 2010; Lim et al., 2013b).

The slip rate on the $\alpha$-th slip system, $\dot{\gamma}^{\alpha}$, is represented as a power-law function of resolved shear stress, $\tau^{\alpha}$, and slip resistance, $g^{\alpha}$ (Hutchinson, 1976):

$$
\dot{\gamma}^{\alpha}=\dot{\gamma}_{0}^{\alpha}\left(\frac{\tau^{\alpha}}{g^{\alpha}}\right)^{1 / m}
$$

where $\dot{\gamma}_{0}^{\alpha}$ is the reference shear rate and $m$ is the rate sensitivity factor. In this work, we adopt commonly-used values of $\dot{\gamma}_{0}^{\alpha}=0.001 \mathrm{~s}^{-1}$ and $m=0.012$ (Bronkhorst et al., 1992; Kalidindi et al., 1992). Note that the value of $m$ used in this study is adopted from studies of FCC metals, such that at $T>T_{c}$ where $\tau^{*}$ can be neglected, the model behaves similar to an FCC model. Such behavior is appropriate in this regime, and deviations to the value of $m$ in this range do not significantly influence the slip behavior by design. In addition, $24\{110\}\langle 111\rangle$ slip systems are used (Table 4), which is a decision supported by many atomistic simulations and experiments (Weinberger et al., 2013a; Carroll et al., 2013). 
The slip resistance, $g^{\alpha}$, can be written as follows (Weinberger et al., 2012):

$$
g^{\alpha}=\min \left(\tau_{\mathrm{EI}}^{* \alpha}, \tau_{\mathrm{LT}}^{* \alpha}\right)+\tau_{\mathrm{obs}}^{\alpha}
$$

where $\tau_{\text {obs }}^{\alpha}$ is the obstacle stress on slip system $\alpha$ and represents the athermal part of the resolved shear stress. Note that the slip resistance, $g$, in Equation (13) is obtained for each slip system at each finite element integration point depending on the local plastic strain rate. On the other hand, it is assumed that the temperature is constant throughout the deformation; thermal effects such as deformation induced heating are neglected. In comparing Equation (13) to Equation (1), $g^{\alpha}$ corresponds to $\tau(T, \dot{\gamma}), \min \left(\tau_{\mathrm{EI}}^{*}, \tau_{\mathrm{LT}}^{*}\right)$ to $\tau^{*}(T, \dot{\gamma})$, and $\tau_{\mathrm{obs}}^{\alpha}$ to $\bar{\tau}$. In this work, we use the $\tau_{L T}^{*}$ derived from the antiparabolic representation of the Peierls potential, Equation (11). Alternatively, other LT models, such as the Eshelby model and the empirical formulation (Equations (7) - (9)), can be implemented in CP-FEM model (Lim et al., 2013a).

The obstacle strength, $\tau_{\mathrm{obs}}^{\alpha}$, represents the strain hardening as a function of the forest dislocation densities as follows (Taylor, 1934; Lee et al., 2010):

$$
\tau_{\mathrm{obs}}^{\alpha}=A \mu b \sqrt{\sum_{\beta=1}^{\mathrm{NS}} \rho^{\beta}}
$$

where $A$ is a material constant usually in the range of $0.3-0.6$ (Wiedersich, 1964), $\mu$ is the shear modulus, $b$ is the magnitude of the Burger's vector, NS is the total number of slip systems, and $\rho^{\beta}$ is the dislocation density in slip system $\beta$. In this work, an average value of 0.4 is used for $A$ (Lim et al., 2011). If it is assumed that initial dislocation densities are identical for all slip systems, then $\tau_{\mathrm{obs}}^{\alpha}(t=0)=\bar{\tau}$ is satisfied. The total dislocation density for $\alpha$-th slip system is obtained by using a standard phenomenological equation as follows (Kocks, 1976):

$$
\dot{\rho}^{\alpha}=\left(\kappa_{1} \sqrt{\sum_{\beta=1}^{\mathrm{NS}} \rho^{\beta}}-\kappa_{2} \rho^{\alpha}\right) \cdot\left|\dot{\gamma}^{\alpha}\right|
$$

where $\kappa_{1}$ and $\kappa_{2}$ are material parameters representing generation and annihilation of dislocations, respectively. $\kappa_{1}$ and $\kappa_{2}$ can be determined from fitting the model to the measured stress-strain curves as shown in Figure 6. Table 5 lists best-fit $\kappa_{1}$ and $\kappa_{2}$. Note that the hardening behavior has 
a secondary importance on yield stress predictions that will be presented in the following sections.

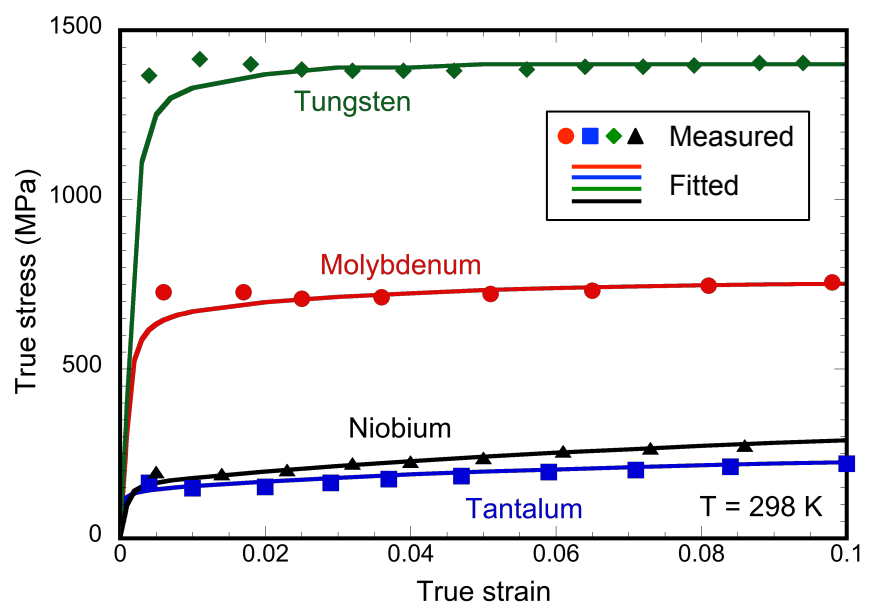

Figure 6: Measured and fitted stress-strain responses of molybdenum, tantalum, tungsten and niobium polycrystals (Hoge and Mukherjee, 1977; Nemat-Nasser and Hori, 1999; Lennon and Ramesh, 2000; Nemat-Nasser and Guo, 2000a).

Table 5: $\kappa_{1}$ and $\kappa_{2}$ used in the simulations for molybdenum, tantalum, tungsten and niobium.

\begin{tabular}{c|cccc}
\hline & Mo & Ta & W & Nb \\
\hline$\kappa_{1}\left(m^{-1}\right)$ & $1.0 \times 10^{6}$ & $1.4 \times 10^{6}$ & $5.0 \times 10^{5}$ & $3.0 \times 10^{6}$ \\
$\kappa_{2}$ & 18 & 14 & 5 & 10 \\
\hline
\end{tabular}

\section{Yield Stress Predictions}

The kink-pair-derived flow rules for four BCC transition metals described in the previous section were implemented into a crystal plasticity code to simulate uniaxial tension of single crystals and polycrystals. The elastic constants, Burger's vector and $\bar{\tau}$ used in the simulations for each material are listed in Table 6.

Single crystal CP-FEM simulations were conducted using a single hexahedral finite element with a crystal orientation of [149] along the tensile axis; this particular orientation was chosen because it is the softest orientation, with the highest degree of resolved shear on the slip plane. The resolved shear stresses from existing experimental data (Hollang et al., 1997; Werner, 1987; Brunner, 2000; Seeger and Holzwarth, 2006) were converted to a uniaxial tensile stress by adding in $\bar{\tau}$ and using a Schmid factor of 0.5. These temperature and strain rate dependent tensile stresses for four BCC 
Table 6: Elastic constants, Burger's vectors and $\bar{\tau}$ for molybdenum, tantalum, tungsten and niobium.

\begin{tabular}{c|ccc|cc}
\hline Materials & $C_{11}(\mathrm{GPa})$ & $C_{12}(\mathrm{GPa})$ & $C_{44}(\mathrm{GPa})$ & $b(\AA)$ & $\bar{\tau}(\mathrm{MPa})$ \\
\hline $\mathrm{Mo}$ & 460 & 176 & 110 & 2.72 & 53 \\
$\mathrm{Ta}$ & 267 & 161 & 82.5 & 2.87 & 27 \\
$\mathrm{~W}$ & 521 & 201 & 160 & 2.74 & 12 \\
$\mathrm{Nb}$ & 246 & 134 & 28.7 & 2.86 & 18 \\
\hline
\end{tabular}

transition metals were compared with predicted yield stresses from CP-FEM simulations using a $0.2 \%$ strain offset criterion. Note that for molybdenum, tantalum and niobium single crystals, flow stresses obtained from Ackermann-Mughrabi cyclic tests were compared with yield stress from tensile test simulations. Given that the rate of hardening in these materials is very low, flow stress obtained from the Ackermann-Mughrabi technique can be assumed to be comparable to the yield stress. In addition, temperature and strain rate dependent $\tau^{*}$ would have no significant effect on pre-deformation. As shown in Figures 7 (a)-(d), CP-FEM simulations of single crystals accurately reproduce temperature and strain rate dependent flow stresses of all four BCC transition metals.

To simulate polycrystal deformation, we used a three-dimensional cubic specimen having 125 cubic grains $(5 \times 5 \times 5)$ with 64 elements per grain (total of 8,000 elements) as shown in Figure 8 (a). Each grain was assigned a random initial crystal orientation from a uniform distribution. Uniaxial tension was simulated with (i) varying temperature at constant strain rates of $10^{-4} \mathrm{~s}^{-1}$ for molybdenum and tantalum and $10^{-3} \mathrm{~s}^{-1}$ for tungsten and niobium, and (ii) varying strain rate at room temperature. Two different strain rates were used in case (i) to facilitate comparison with available experimental data. Predicted yield stresses were compared with various experimental data from both the literature and our own tensile tests.

Figures 9 and 10 show measured and predicted yield stresses versus temperature and strain rate, respectively, in polycrystalline materials. CP-FEM simulations of yield stresses (red circles) exhibit the same trends observed in the experimental data from various sources (Mathew and Murty, 1999; ASM, 1990; Briggs and Campbell, 1972; Hoge and Mukherjee, 1977; Park et al., 2011; Lennon and Ramesh, 2000; Raffo, 1968; Nemat-Nasser and Guo, 2000a; Davidson et al., 1966; Voyiadjis and Abed, 2006; Adams and Iannucci, 1961; Chen and Gray, 1996). This agreement suggests that the thermal part of the flow stresses in polycrystals is adequately predicted using the CP-FEM 

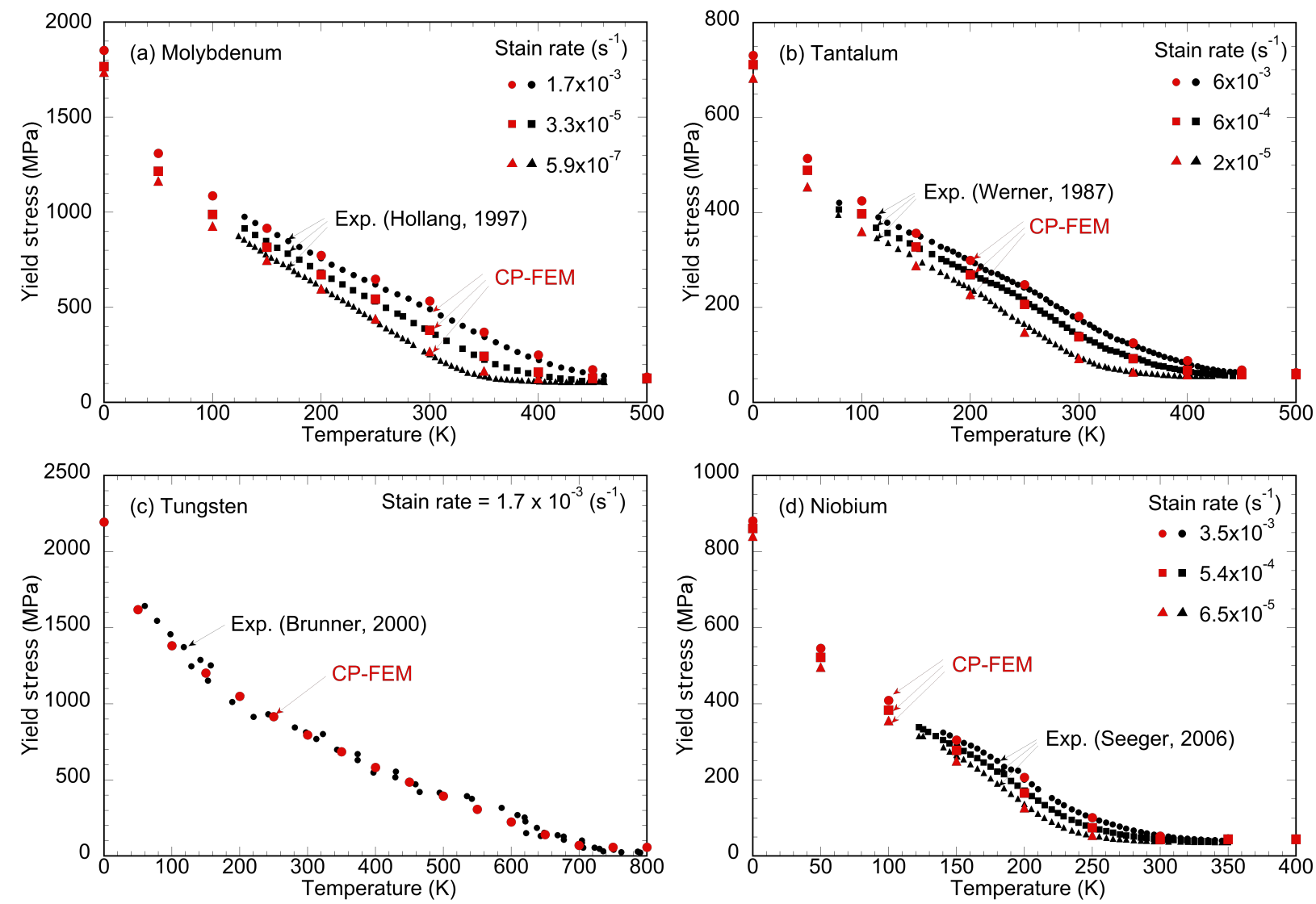

Figure 7: Measured and predicted yield stresses versus temperature and strain rates for [149] single crystals (a) molybdenum, (b) tantalum, (c) tungsten and (d) niobium.

simulation informed by the single crystal data.

In the case of molybdenum, tantalum and tungsten (Figures 9 (a)-(c) and 10 (a)-(c)), the simulated and measured data agree well in magnitude. However, for niobium, the simulated response of the polycrystals (red diamonds in Figures 9 (d) and 10 (d)) differs in magnitude from the experimental data. This is likely due to the calibration of the athermal contribution to the flow stress, $\bar{\tau}$, which does not depend significantly on temperature or strain rate, but instead is sensitive to the grain size, initial dislocation density, impurities, heat treatments and prior deformation. Thus, changes to $\bar{\tau}$ serve only to shift the data up or down in magnitude, and not to change the trends in the data. Note that while the tungsten single crystal data were obtained from uniaxial tension tests (Brunner, 2000), the kink-pair constants for molybdenum, tantalum and niobium single crystals were calibrated to data from cyclic tests using the Ackermann-Mughrabi technique (Seeger, 1981; Werner, 1987; Hollang et al., 1997, 2001; Seeger and Holzwarth, 2006). While this calibra- 


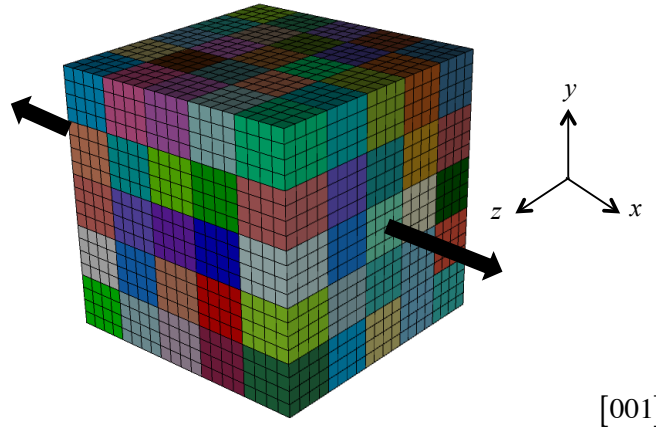

(a)

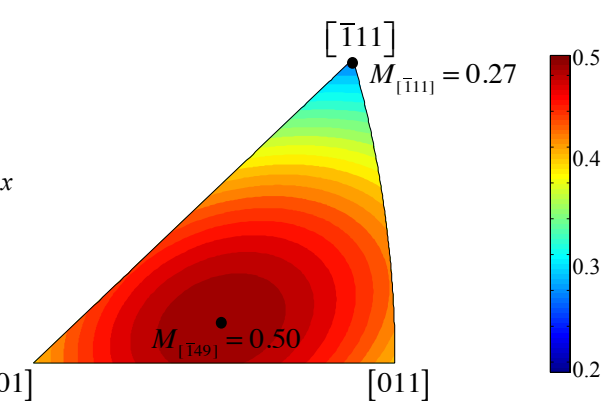

(b)

Figure 8: (a) Schematic of an idealized 3D grain assembly used for polycrystal simulations, and (b) the maximum Schmid factors of $\{110\}\langle 111\rangle$ slip systems within the stereographic triangle. Single crystals oriented for [111] and [149] loading directions have the maximum and minimum values of 0.50 and 0.27 .

tion appears to be acceptable for molybdenum and tantalum, the same appears to not be true of niobium. The red circles in Figures 9 (d) and 10 (d) represent simulations of niobium polycrystals with a revised value of $\bar{\tau}=60 \mathrm{MPa}$ calibrated to data for high temperature and low strain rate (Nemat-Nasser and Guo, 2000a), and this re-calibration of the athermal contribution to the flow stress results in much better agreement for the polycrystal predictions in niobium.

Figure 8 (b) shows the maximum Schmid factors of $\{110\}\langle 111\rangle$ slip system within the stereographic triangle. Single crystals oriented for [111] and [149] tension have the minimum and maximum Schmid factors of 0.27 and 0.50, respectively. Thus, [111] and [149] single crystals provide yield stresses of the hardest and softest orientations, respectively. Therefore, these cases (dashed lines in Figures 9 and 10) provide an estimate of the upper and lower limits of strength in polycrystals (neglecting other microstructural effects such as hardening due to grain boundaries, constraint from neighboring grains and non-Schmid effects). As shown in Figures 9 (a)-(d) and 10 (a)-(d), various experimental data from the literature fall largely within this simple depiction of upper and lower limits. This is especially encouraging since the model calibration was performed using data from only single crystal tests.

\section{Polycrystal Continuum-Scale Models}

Several continuum-scale constitutive models exist for describing the flow stress of BCC metals at high temperatures and strain rates (Johnson and Cook, 1983, 1985; Zerilli and Armstrong, 1987; Follansbee and Kocks, 1988). These models are generally fit to experimental data of polycrystals 

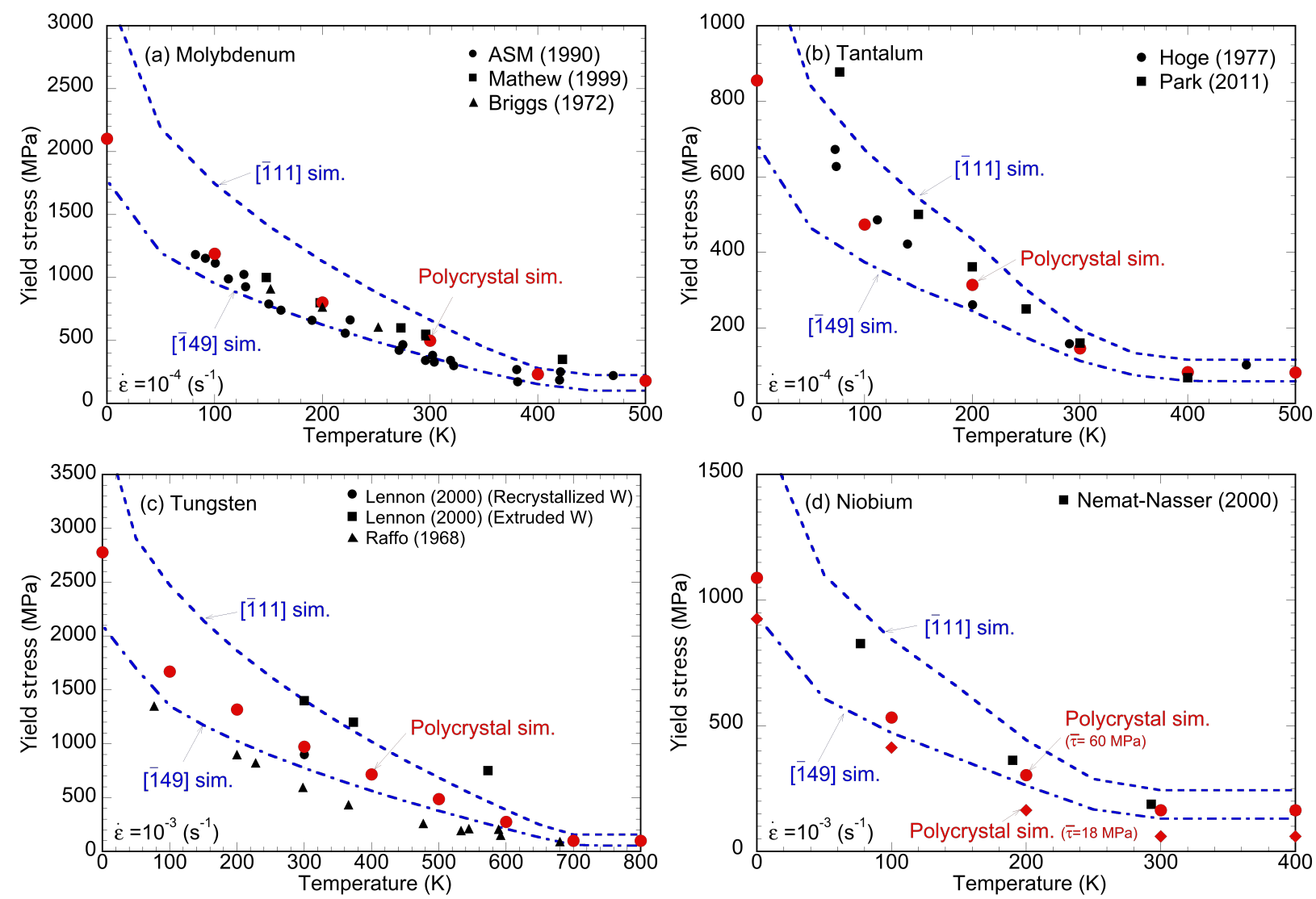

Figure 9: A plot of measured and predicted yield stresses of polycrystals at different temperatures. (a) molybdenum, (b) tantalum, (c) tungsten and (d) niobium.

to obtain material constants and reproduce measured flow behavior. Using an analogous approach, we fit some of these constitutive models to CP-FEM polycrystal simulation results in an attempt not only to investigate how well these models reproduce temperature and strain rate dependent yield behavior of four BCC transition metals, but also to construct a simple polycrystal constitutive model that can be efficiently used in other applications, e.g. large scale metal forming analysis or shock applications, without requiring extremely large and expensive CP-FEM calculations.

In this work, three continuum/ macroscale constitutive models are considered: Johnson-Cook (JC) (Johnson and Cook, 1983, 1985), Zerilli-Armstrong (ZA) (Zerilli and Armstrong, 1987) and Mechanical Threshold Stress (MTS) (Follansbee and Kocks, 1988). If we assume that the yield stress, $\sigma_{y}$, is obtained at $\epsilon_{p}=0$, the JC, ZA and MTS models have the following forms:

$$
\sigma_{y}^{J C}=A(1+C \cdot \ln \dot{\epsilon})\left(1-T^{* m}\right)
$$



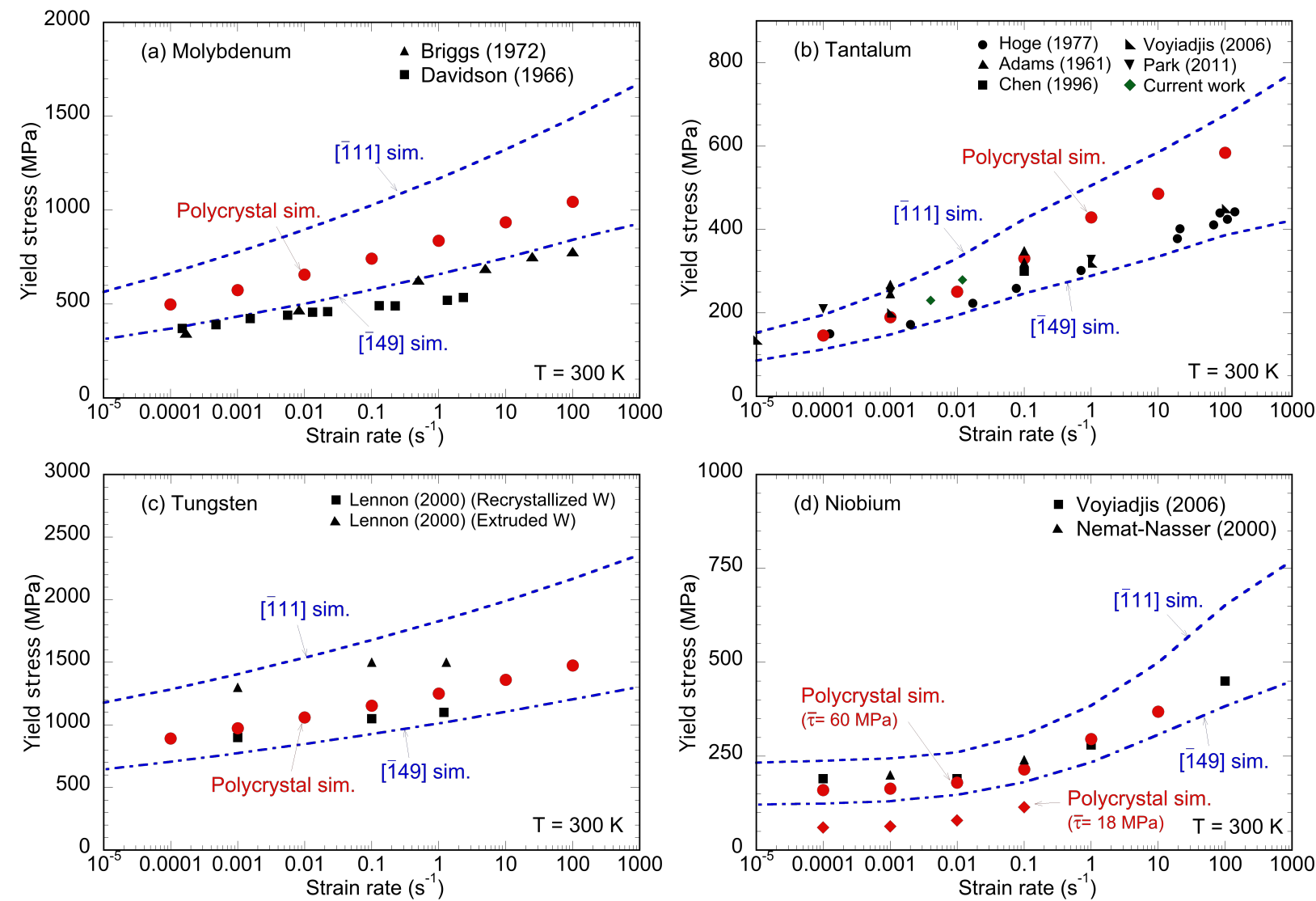

Figure 10: A plot of measured and predicted yield stresses of polycrystals at different strain rates at $300 \mathrm{~K}$. (a) molybdenum, (b) tantalum, (c) tungsten and (d) niobium.

$$
\begin{gathered}
\sigma_{y}^{Z A}=C_{0}+C_{1} \exp \left(-C_{3} T+C_{4} T \ln \dot{\epsilon}\right) \\
\sigma_{y}^{M T S}=\sigma_{0}+\hat{\sigma}\left(1-\left(-\frac{k_{\mathrm{B}}}{G_{0}} T \ln \frac{\dot{\epsilon}}{\dot{\epsilon}_{0}}\right)^{1 / q}\right)^{1 / p}
\end{gathered}
$$

where $\dot{\epsilon}$ is the strain rate, $T^{*}$ is $\left(T-T_{\text {room }}\right) /\left(T_{\text {melt }}-T_{\text {room }}\right), G_{0}$ is the activation energy per atom to overcome obstacles, $\dot{\epsilon}_{0}$ is the reference strain rate, and the $p$ and $q$ in Equation (18) represent parameters that determine the shape of the energy barrier profile such that $0 \leq p \leq 1$ and $1 \leq q \leq 2$ (Kocks et al., 1975). Note that $\dot{\epsilon}$ in Equations (16)-(18) represent the macroscopic strain rates of polycrystals while $\dot{\gamma}$ used in the EI and LT models denote resolved shear strain rates of single crystals. The EI model and the LT model adopting antiparabolic Peierls potential have the same form as that of the MTS model (compare Equations (4) and (11) to Equation (18)) with $p=0.5$, 
$q=1$ and $p=1, q=2$ in two different temperature/ stress regimes, respectively. In contrast, most MTS models are generally fit with arbitrarily chosen values of $p$ and $q$ for all temperature and strain rates (Nemat-Nasser et al., 1999; Cheng et al., 2001; Park et al., 2011; Nemat-Nasser and Isaacs, 1997; Nemat-Nasser and Kapoor, 2001; Nemat-Nasser and Guo, 2000a). Despite the similar functional forms of the EI/ LT models with the MTS model, the EI and LT models are derived from physics of dislocation motion via kink-pair nucleation while the MTS model is a generic phenomenological model of thermally activated flow.

The JC, ZA and MTS models require three, four and six material parameters, respectively, that are obtained from fitting to experimental data. In order to parameterize these material constants in Equations (16) - (18), yield stresses predicted by polycrystal CP-FEM simulations (red circles in Figures 9 and 10) are fit to each constitutive equation using a least-squares procedure. For the JC model, we use $T^{*}=T / T_{\text {melt }}$ to capture yield stresses below room temperature, avoiding negative $T^{*}$. For the ZA and MTS models, the athermal part of the yield stress, $C_{0}$ and $\sigma_{0}$, are determined from the yield stresses at high temperature, i.e. $181 \mathrm{MPa}, 82 \mathrm{MPa}, 99 \mathrm{MPa}$ and 164 MPa for molybdenum, tantalum, tungsten and niobium, respectively. Previous studies adopting the MTS model usually assume constant values of $p$ and $q$ in the range of $0<p \leq 1$ and $1 \leq q \leq 2$, and estimate $\dot{\epsilon}_{0}$ from $\dot{\epsilon}_{0}=b \rho_{m} w_{0} d$ where $\rho_{m}$ is the mobile dislocation density, $w_{0}$ is the attempt frequency and $d$ is the lattice spacing. In this work, all six parameters in the MTS model, $p, q$, $\dot{\epsilon}_{0}, \sigma_{0}, \hat{\sigma}$ and $G_{0}$, are determined directly from the fit. The MTS model in Equation (18) is only applicable when $\dot{\epsilon} \leq \dot{\epsilon}_{0}$ and $-\frac{k_{\mathrm{B}}}{G_{0}} T \ln \frac{\dot{\epsilon}}{\dot{\epsilon}_{0}} \leq 1$ are satisfied. However, at the very high temperature/ low strain rate regime, it is numerically possible to have $-\frac{k_{\mathrm{B}}}{G_{0}} T \ln \frac{\dot{\epsilon}}{\dot{\epsilon}_{0}}$ larger than 1 . In this case, we assume that there is no thermal/ strain rate dependent stress contributions and use $\sigma_{0}$ to represent the yield stress.

Figures 11 and 12 compare yield stresses fit to CP-FEM simulations using three constitutive formulations. For all four BCC metals, the yield stresses are accurately obtained by the MTS and ZA models, while the JC model produced a relatively poor-quality fit, consistent with the earlier work on tantalum and tantalum-tungsten alloys (Chen and Gray, 1996). Note that the JC model has a linear relationship between the yield stress and the logarithm of strain rate, and thus is not easily able to reproduce temperature and strain rate dependent flow stresses simultaneously. Also note that the predicted yield stresses saturate at high temperature/ low strain rate regimes for 

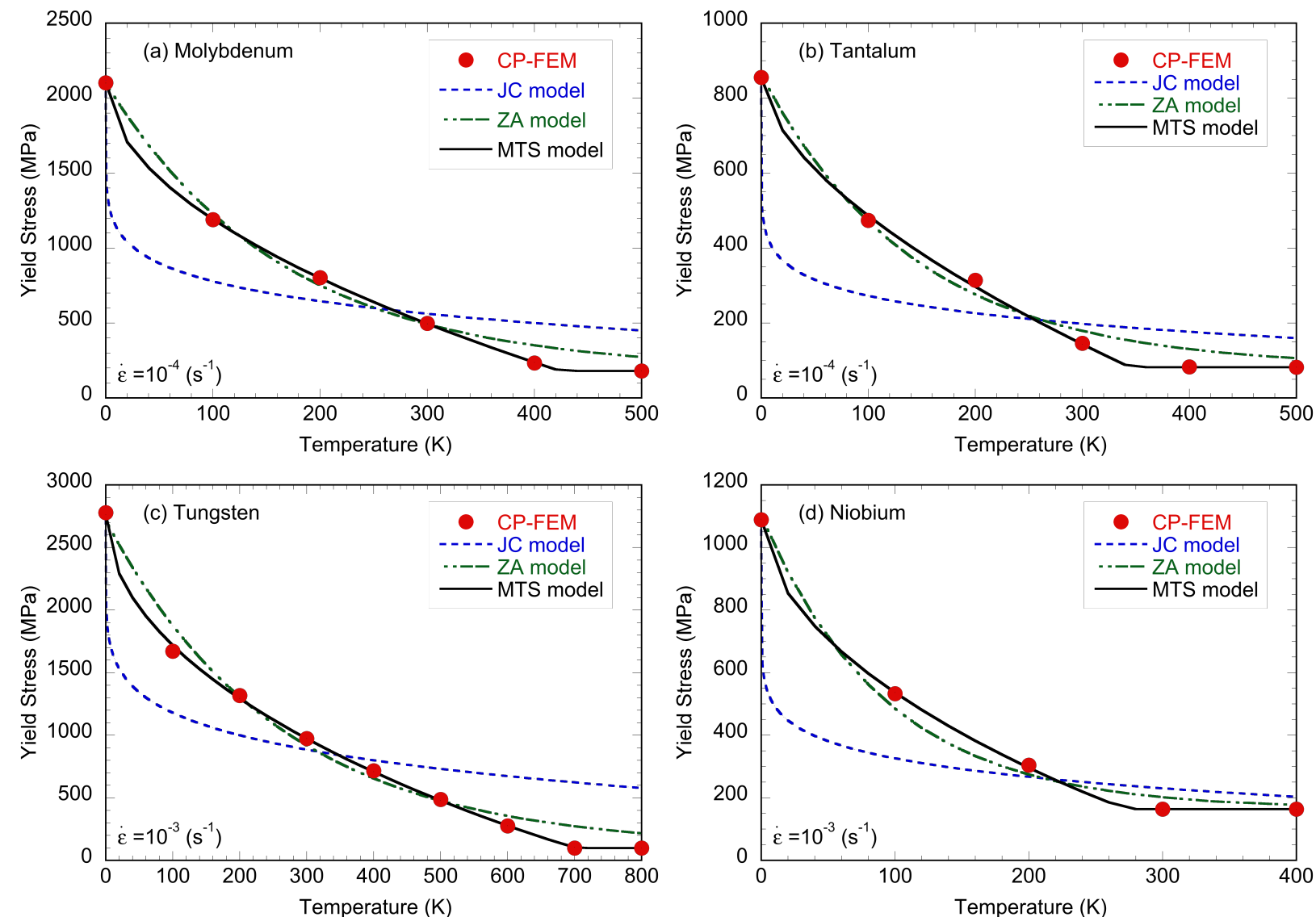

Figure 11: A plot of yield stresses fit to CP-FEM simulations using the JC, ZA and MTS models for different temperature. (a) molybdenum, (b) tantalum, (c) tungsten and (d) niobium.

the MTS model where $-\frac{k_{\mathrm{B}}}{G_{0}} T \ln \frac{\dot{\epsilon}}{\dot{\epsilon}_{0}}$ is larger than 1 and the yield stress is equal to $\sigma_{0}$, consistent with CP-FEM predictions. Table 7 lists the standard deviations of yield stresses between CP-FEM predictions and three continuum fits. It is clearly shown that the temperature and strain rate dependent flow stress is most accurately reproduced using the MTS model. Table 8 lists best-fit material parameters for the three models. For the MTS model, the best-fit $p$ and $q$ obey $0<p \leq 1$ and $1 \leq q \leq 2$ (Kocks et al., 1975) and the best-fit $G_{0}$ ranges from $0.49 \mathrm{eV}$ to $1.42 \mathrm{eV}$, the same order of magnitude as $2 H_{k}$ obtained from the kink-pair analysis (Table 1 ). $C_{1}$ and $\hat{\sigma}$ representing the thermal part of the flow stress at $0 \mathrm{~K}$ in the ZA and MTS models show good agreement.

In comparing the kink-pair model and the MTS model, $\dot{\gamma}_{0}$ corresponds to $\dot{\epsilon}_{0}, 2 H_{k}$ to $G_{0}$ and $\tau_{p}$ to $\hat{\sigma}$. For BCC polycrystals, it can be estimated that $G_{0}=2 H_{k}, \dot{\epsilon}_{0}=M \dot{\gamma}_{0}$ and $\hat{\sigma}=M \tau_{p}$ where $M$ is the average Taylor factor (Taylor, 1938). Thus, the MTS model can be fit to CP-FEM 

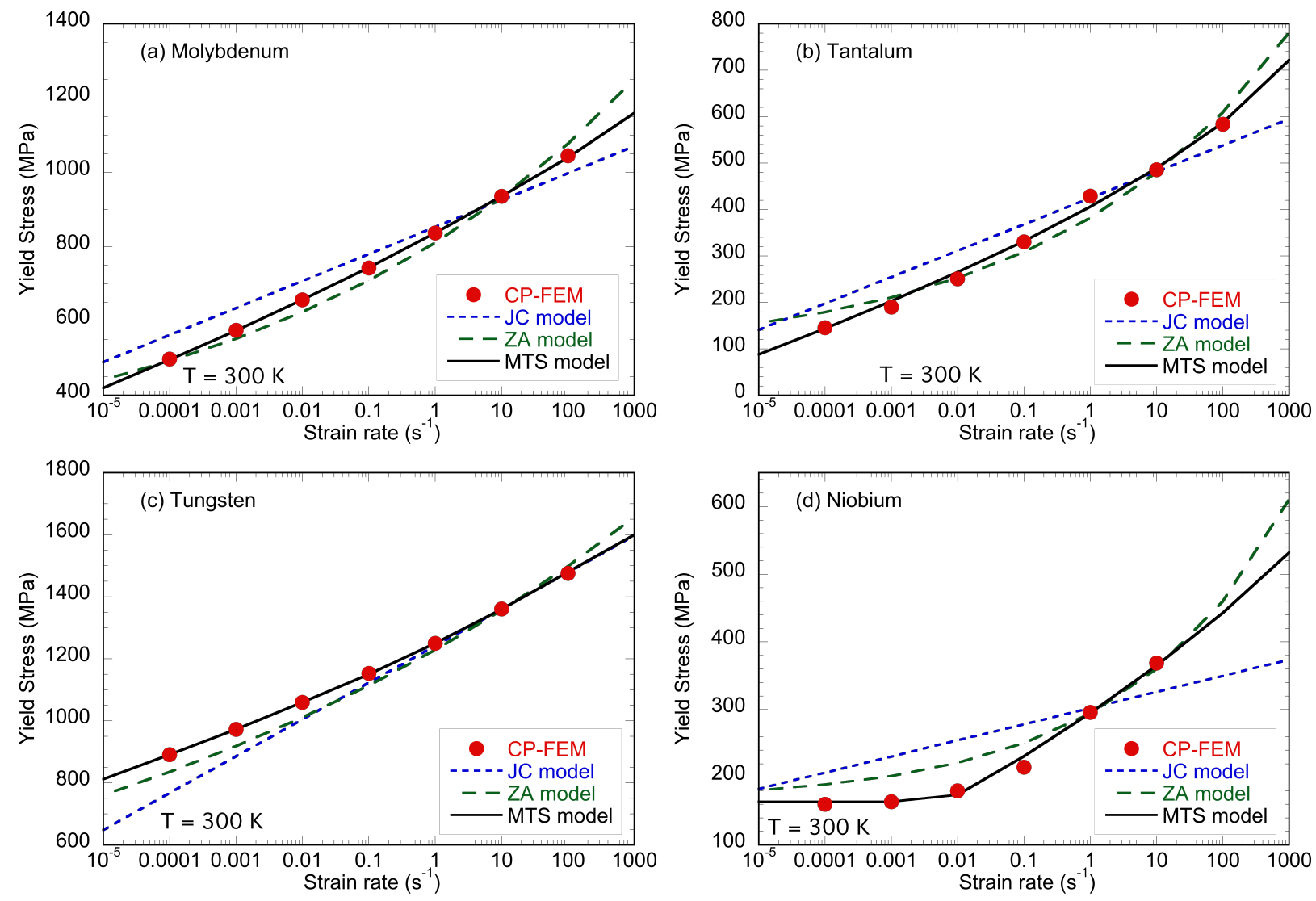

Figure 12: A plot of yield stresses fit to CP-FEM simulations using the JC, ZA and MTS models for different strain rates at $300 \mathrm{~K}$. (a) molybdenum, (b) tantalum, (c) tungsten and (d) niobium.

polycrystal predictions with material parameters informed from the kink-pair analysis. Values in parentheses in Tables 7 and 8 represent values obtained using $G_{0}=2 H_{k}$ and $\dot{\epsilon}_{0}=M \dot{\gamma}_{0}$, obtained from the kink-pair analyses (Table 1) while $\hat{\sigma}, p$ and $q$ values are fit to CP-FEM predictions.

It is shown in Table 7 that the MTS model using material parameters informed from the kinkpair model reproduces yield behaviors relatively well, but not as accurate as the MTS model with all parameters fit to the data. Using material parameters informed from kink-pair theory still provides better predictions than either the JC or ZA models; furthermore, this approach has the advantage of being more physically based. As listed in Table 8, the best-fit $\hat{\sigma}$ obtained from two MTS fits agrees well while the other material parameters, $G_{0}, \dot{\epsilon}_{0}, p$ and $q$, do not show obvious correlations. This implies that the continuum-scale model is able to reproduce the observed macroscopic polycrystalline behavior but it is unable to predict the single crystal, and hence dislocation-scale, properties. Thus, the multi-scale formulation provided here allows one to propagate lower-scale 
Table 7: Standard deviations of yield stresses between CP-FEM predictions and continuum fits using the JC, ZA and MTS models (unit: MPa). Values in parentheses in MTS model represent fitting results using $G_{0}=2 H_{k}$ and $\dot{\epsilon}_{0}=M \dot{\gamma}_{0}$, obtained from the kink-pair analysis.

\begin{tabular}{c|ccc}
\hline & JC model & ZA model & MTS model \\
\hline Mo & 165.4 & 48.9 & $2.1(45.6)$ \\
$\mathrm{Ta}$ & 78.3 & 28.2 & $10.2(48.0)$ \\
$\mathrm{W}$ & 242.1 & 82.0 & $14.2(51.4)$ \\
$\mathrm{Nb}$ & 78.7 & 30.4 & $6.0(23.7)$ \\
\hline Avg. & 141.1 & 47.4 & $8.1(42.2)$ \\
\hline
\end{tabular}

Table 8: Best-fit constants used for the JC, ZA and MTS models. Values in parentheses in MTS model represent best-fit constants using $G_{0}=2 H_{k}$ and $\dot{\epsilon}_{0}=M \dot{\gamma}_{0}$, obtained from the kink-pair analysis.

\begin{tabular}{|c|c|c|c|c|c|}
\hline Models & Parameters & Mo & $\mathrm{Ta}$ & W & $\mathrm{Nb}$ \\
\hline \multirow{3}{*}{$\mathrm{JC}$} & $A(\mathrm{MPa})$ & 3214 & 1848 & 4028 & 1697 \\
\hline & $C$ & 0.037 & 0.058 & 0.040 & 0.049 \\
\hline & $m$ & 0.136 & 0.109 & 0.149 & 0.085 \\
\hline \multirow{3}{*}{$\mathrm{ZA}$} & $C_{1}(\mathrm{MPa})$ & 1918 & 779 & 2617 & 937 \\
\hline & $C_{3}\left(K^{-1}\right)$ & $3.71 \times 10^{-3}$ & $3.18 \times 10^{-3}$ & $2.80 \times 10^{-3}$ & $6.58 \times 10^{-3}$ \\
\hline & $C_{4}\left(K^{-1} \cdot s^{-1}\right)$ & $2.55 \times 10^{-4}$ & $4.08 \times 10^{-4}$ & $1.55 \times 10^{-4}$ & $5.95 \times 10^{-4}$ \\
\hline \multirow{5}{*}{ MTS } & $G_{0}(\mathrm{eV})$ & $0.91(1.27)$ & $0.51(0.85)$ & $1.42(2.06)$ & $0.49(0.68)$ \\
\hline & $\dot{\epsilon}_{0}\left(s^{-1}\right)$ & $6.8 \times 10^{6}\left(1.1 \times 10^{10}\right)$ & $2.8 \times 10^{3}\left(9.1 \times 10^{6}\right)$ & $1.5 \times 10^{7}\left(1.1 \times 10^{11}\right)$ & $1.20 \times 10^{6}\left(3.5 \times 10^{8}\right)$ \\
\hline & $\hat{\sigma}(\mathrm{MPa})$ & 1921(1915) & $772(767)$ & $2664(2673)$ & $925(924)$ \\
\hline & $p$ & $1.00(0.85)$ & $1.00(0.69)$ & $0.95(0.84)$ & $0.96(0.97)$ \\
\hline & $q$ & $1.93(1.56)$ & $1.67(1.00)$ & $2.00(1.76)$ & $1.86(1.98)$ \\
\hline
\end{tabular}

information up to the continuum, but demonstrates that larger length scale models cannot provide insight into the physics at lower length scales, which is perhaps not surprising.

\section{Conclusions}

In this work, we developed a CP-FEM model by calibrating kink-pair theory to flow stress measurements in single crystal molybdenum, tantalum, tungsten and niobium. The CP-FEM model was then used to calibrate continuum scale plasticity models to the physically-based, polycrystal predictions. This involved fitting analytical expressions of the flow stresses, $\tau^{*}$, as a function of temperature and strain rate. The shape of the Peierls potential has little effect on the fit at 
moderate temperatures, but is important at low temperatures.

The BCC crystal plasticity finite element model developed herein from dislocation kink-pair theory accurately captures temperature and strain rate dependent yield stresses of single crystal molybdenum, tantalum, tungsten and niobium. The thermal contribution to the flow stress of a simulated polycrystalline aggregate agrees well with various experimental results, and falls within

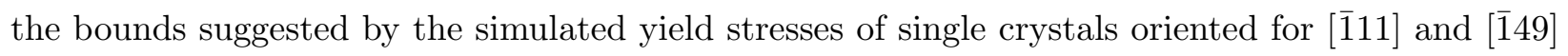
tensile loading. The athermal contribution to the flow stress depends on various factors - e.g. previous loading history or heat treatments that can alter grain size, impurity distributions and forest dislocations - and thus is best obtained from high temperature and low strain rate tensile data.

Three continuum-scale constitutive formulations that model flow stresses at high temperatures and strain rates were fit to simulated yield stresses of polycrystal molybdenum, tantalum, tungsten and niobium. These models, when calibrated to CP-FEM simulations, can be used to replicate yield behavior of polycrystals without the need to perform expensive, direct CP-FEM simulations. The Mechanical Threshold Stress model was found to reproduce the simulated yield stresses of polycrystal BCC metals most accurately. The framework developed in this work provides a way to connect dislocation physics to grain scale deformation, and in turn to conventional continuum models of yield behavior at high temperatures and strain rates.

\section{Acknowledgement}

Sandia National Laboratories is a multi-program laboratory managed and operated by Sandia Corporation, a wholly owned subsidiary of Lockheed Martin Corporation, for the U.S. Department of Energys National Nuclear Security Administration under contract DE-AC04-94AL85000.

\section{References}

Ackermann, F., Mughrabi, H., Seeger, A., 1983. Temperature- and strain-rate dependence of the flow stress of ultrapure niobium single crystals in cyclic deformation. Acta. Metall. 31, 1353-1366.

Adams, M.A., Iannucci, A., 1961. The mechanical properties of tantalum with special reference to the ductile-brittle transition. ASD Technical Report, 61-203. 
Argon, A., 2008. Strengthening mechanisms in crystal plasticity. Oxford University Press.

Asaro, R.J., 1983. Micromechanics of crystals and polycrystals. Adv. Appl. Mech. 23, 1-115.

Asaro, R.J., Needleman, A., 1985. Texture development and strain hardening in rate dependent polycrystals. Acta Metall. 33, 923-953.

ASM, 1990. ASM Handbook Volume 2: Properties and Selection : Nonferrous Alloys and SpecialPurpose Materials. volume 2.

Biffle, J.H., 1987. A three-dimensional finite element computer program for the nonlinear quasistatic response of solids with the conjugate gradient method, SAND87-1305. Sandia National Laboratories.

Briggs, T.L., Campbell, J.D., 1972. The effect of strain rate and temperature on the yield and flow of polycrystalline niobium and molybdenum. Acta Metallurgica 20, 711-724.

Bronkhorst, C.A., Kalidindi, S.R., Anand, L., 1992. Polycrystalline plasticity and the evolution of crystallographic texture in fcc metals. Philos. Trans. Roy. Soc. London A 341, 443-477.

Brunner, D., 2000. Comparison of flow-stress measurements on high-purity tungsten single crystals with the kink-pair theory. Mater. Trans. JIM 41, 152-160.

Brunner, D., Diehl, J., 1991. Strain-rate and temperature dependence of the tensile flow stress of high-purity $\alpha$-iron above $250 \mathrm{k}$ (regime i) studied by means of stress-relaxation tests. Phys. Status Solidi A 124, 155-170.

Butt, M.Z., 2007. Kinetics of flow stress in crystals with high intrinsic lattice friction. Philos. Mag. $87,3595-3614$.

Caillard, D., Martin, J.L., 2003. Thermally activated mechanisms in crystal plasticity. Pergamon.

Carroll, J.D., Clark, B.G., Buchheit, T.E., Boyce, B.L., Weinberger, C.R., 2013. An experimental statistical analysis of stress projection factors in bcc tantalum. Mat. Sci. Eng. A 581, 108-118.

Celli, V., Kabler, M., Ninomiya, T., Thomson, R., 1963. Theory of dislocation mobility in semiconductors. Phys. Rev. 131, 58-72. 
Chen, S.R., Gray, G.T., 1996. Constitutive behavior of tantalum and tantalum-tungsten alloys. Met. Mater. Trans. A 27, 2994-3005.

Cheng, J., Nemat-Nasser, S., Guo, W., 2001. A unified constitutive model for strain-rate and temperature dependent behavior of molybdenum. Mech. Mat. 33, 603-616.

Christian, J.W., 1983. Some surprising features of the plastic deformation of body-centered-cubic metals and alloys. Met. Trans. A 14A, 1237-1256.

Davidson, D.L., Lindholm, U.S., Yeakley, L.M., 1966. The deformation behavior of high purity polycrystalline iron and single crystal molybdenum as a function of strain rate at 300k. Acta Metallurgica 14, 702-710.

Dawson, P.R., 2000. Computational crystal plasticity. Int. J. Sol. Struct. 37, 115-130.

Dingreville, R., Battaile, C.C., Brewer, L.N., Holm, E.A., Boyce, B.L., 2010. The effect of microstructural representation on simulations of microplastic ratcheting. Int. J. Plasticity 21, 617633.

Dorn, J.E., Rajnak, S., 1964. Nucleation of kink pairs + peierls mechanism of plastic deformation. Transactions of the Metallurgical Society of AIME 230, 1052-1064.

Duesbery, M.S., Vitek, V., 1998. Plastic anisotropy in B.C.C. transition metals. Acta. Mater. 46, $1481-1492$.

Eshelby, J.D., 1962. The interactions of kinks with elastic waves. Proc. R.l Soc. Lond. A 266, $222-246$.

Follansbee, P.S., Kocks, U.F., 1988. A constitutive description of the deformation of copper based on the use of the mechanical threshold stress as an internal state variable. Acta metall. 1, 81-93.

Gröger, R., Bailey, A., Vitek, V., 2008. Multiscale modeling of plastic deformation of molybedenum and tungsten: I. Atomistic studies of the core structure and glide of $1 / 2\langle 111\rangle$ screw dislocations at 0 k. Acta. Mater. 56, 5401-5411.

Guyot, M., Dorn, J.E., 1964. A critical review of the peierls mechanism. Canadian Journal of Physics 43, 983-1016. 
Hill, R., Rice, J.R., 1972. Constitutive analysis of elastic plastic crystals at arbitrary strain. J. Mech. Phys. Solids 20, 401-413.

Hirth, J.P., Lothe, J., 1982. Theory of Dislocations. Krieger.

Hoge, K.G., Mukherjee, A.K., 1977. The temperature and strain rate dependence of the flow stress of tantalum. J. Mat. Sci. 12, 1666-1672.

Hollang, L., Brunner, D., Seeger, A., 2001. Work hardening and flow stress of ultrapure molybdenum single crystals. Mater. Sci. Eng. A 319, 233-236.

Hollang, L., Hommel, M., Seeger, A., 1997. The flow stress of ultra-high-purity molybdenum single crystals. Phys. Status Solidi A 160, 329-354.

Hutchinson, J.W., 1976. Bounds and self-consistent estimates for creep of polycrystalline materials. Proc. R. Soc. Lond. A 348, 101-127.

Ito, K., Vitek, V., 2001. Atomistic study of non-Schmid effects in the plastic yielding of bcc metals. Philos. Mag. A 81, 1387-1407.

Johnson, G.R., Cook, W.H., 1985. Fracture characteristics of three metals subjected to various strains, strain rates, temperatures and pressures. Eng. Fract. Mech. 21, 31-48.

Johnson, G.R., Cook, W.J., 1983. A constitutive model and data for metals subjected to large strains, high strain rates and high temperatures. Seventh International Symposium on Ballistics, The Hague, The Netherlands , 541-547.

Kalidindi, S.R., Bronkhorst, C.A., Anand, L., 1992. Crystallographic texture evolution in bulk deformation processing of fcc metals. J. Mech. Phys. Solids 40, 537-569.

Kocks, U.F., 1976. Laws for work-hardening and low-temperature creep. J. Eng. Mater. Tech., ASME 98, 76-85.

Kocks, U.F., Argon, A.S., Ashby, M.F., 1975. Thermodynamics and kinetics of slip. Progress in Materials Science 19, 1-289.

Lee, E.H., 1969. Elastic-plastic deformation at finite strains. Appl. Mech. 36, 1-6. 
Lee, M.G., Lim, H., Kim, J.H., Adams, B.L., Wagoner, R.H., 2010. A dislocation density-based single crystal constitutive equation. Int. J. Plasticity 26, 925-938.

Lennon, A.M., Ramesh, K.T., 2000. The thermoviscoplastic response of polycrystalline tungsten in compression. Mat. Sci. Eng. A 276, 9-21.

Lim, H., Battaile, C.C., Carroll, J.D., Buchheit, T.E., Boyce, B.L., Weinberger, C.R., 2013a. Multi-scale modeling of bcc transition metals. Sandia Report Submitted.

Lim, H., Lee, M.G., Kim, J.H., Adams, B.L., Wagoner, R.H., 2011. Simulation of polycrystal deformation with grain and grain boundary effects. Int. J. Plasticity 27, 1328-1354.

Lim, H., Weinberger, C.R., Battaile, C.C., Buchheit, T.E., 2013b. Application of generalized nonschmid yield law to low temperature plasticity in bcc transition metals. Model. Simul. Mater. Sci. Eng. 21, 045015.

Mathew, M.D., Murty, K.L., 1999. Non-destructive studies on tensile and fracture properties of molybdenum at low temperatures (148 to 423 k). J. Mat. Sci. 34, 1497-1503.

Nemat-Nasser, S., Guo, W., 2000a. Flow stress of commercially pure niobium over a broad range of temperatures and strain rates. Mater. Sci. Eng. A 284, 202-210.

Nemat-Nasser, S., Guo, W., 2000b. High strain-rate response of commercially pure vanadium. Mech. Mat. 32, 243-260.

Nemat-Nasser, S., Guo, W., Liu, M., 1999. Experimentally-based micromechanical modeling of dynamic response of molybdenum. Scripta Materialia 40, 859-872.

Nemat-Nasser, S., Hori, M., 1999. Micromechanics: Overall properties of heterogeneous materials. North-Holland Publishers.

Nemat-Nasser, S., Isaacs, J.B., 1997. Direct measurement of isothermal flow stress of metals at elevated temperatures and high strain rates with application to ta and ta-w alloys. Acta mater. $45,907-919$.

Nemat-Nasser, S., Kapoor, R., 2001. Deformation behavior of tantalum and a tantalum tungsten alloy. Int. J. Plasticity 17, 1351-1366. 
Park, C.H., Hong, S.G., Lee, C.S., 2011. A unified constitutive model for quasi-static flow responses of pure ta and ta-w alloys. Mat. Sci. Eng. A 528, 1154-1161.

Peirce, D., Asaro, R.J., Needleman, A., 1982. An analysis of nonuniform and localized deformation in ductile single crystals. Acta Metall. 30, 1087-1119.

Raffo, P.L., 1968. Yielding and fracture in tungsten and tungsten-rhenium alloys. NASA Technical Note NANA TN D-4567.

Rice, J.R., 1971. Inelastic constitutive relations for solids, an internal-variable theory and its application to metal plasticity. J. Mech. Phys. Solids 19, 433-455.

Seeger, A., 1981. The temperature and strain-rate dependence of the flow stress of body-centered cubic metals: A theory based on kink-kink interactions. Z. Metallikd 72, 369-380.

Seeger, A., 1995. The flow stress of high-purity refractory body-centered cubic metals and its modification by atomic defects. Journal de Physique IV 5, C7-45-C7-65.

Seeger, A., 2001. Why anomalous slip in body-centred cubic metals? Mater. Sci. Eng. A 319-321, $254-260$.

Seeger, A., Holzwarth, U., 2006. Slip planes and kink properties of screw dislocations in high-purity niobium. Philos. Mag. 86, 3861-3892.

Taylor, G., 1992. Thermally-activated deformation of BCC metals and alloys. Prog. Mater. Sci. $36,29-61$.

Taylor, G.I., 1934. The mechanism of plastic deformation of crystals. part i. theoretical. Proc. Roy. Soc. A $165,362-387$.

Taylor, G.I., 1938. Plastic strain in metals. J. Inst. Metals 62, 307-324.

Voyiadjis, G.Z., Abed, F.H., 2006. A coupled temperature and strain rate dependent yield function for dynamic deformations of bcc metals. Int. J. Plasticity 22, 1398-1431.

Weinberger, C.R., Battaile, C.C., Buchheit, T.E., Holm, E.A., 2012. Incorporating atomistic models of lattice friction into bcc crystal plasticity models. Int. J. Plasticity 37, 16-30. 
Weinberger, C.R., Boyce, B.L., Battaile, C.C., 2013a. Slip planes in bcc transition metals. Int. Mater. Rev. 58, 296-314.

Weinberger, C.R., Tucker, G.J., Foiles, S., 2013b. The peierls potential of screw dislocations in bcc transition metals: predictions form density functional theory. Phys. Rev. B 87, 054114.

Werner, M., 1987. Temperature and strain-rate dependence of the flow stress of ultrapure tantalum single crystal. Phys. Status Solidi A 104, 63-78.

Werner, M., Seeger, A., 1988. Temperature and strain-rate dependence of the flow stress of bcc transition metal crystals in cyclic deformation, in: Kettun, P., Lepistö, T., Lehtonen, M. (Eds.), Proc. 8th International Conference on the strength of Metals and Alloys, Pergamon Press. pp. $173-178$.

Wiedersich, H., 1964. Hardening mechanisms and the theory of deformation. J. Met. 16, 425-430.

Zerilli, F.J., Armstrong, R.W., 1987. Dislocation-mechanics-based constitutive relations for material dynamics calculations. J. Appl. Phys. 61, 1816-1825.

Zerilli, F.J., Armstrong, R.W., 1990. Description of tantalum deformation behavior by dislocation mechanics based constitutive relations. J. Appl. Phys. 68, 1580-1591. 